\title{
C-MRS Builds on Past Efforts to Form New Society
}

\section{New Goals and Activities Planned}

In China, efforts to foster scientific and organizing activities in materials science began back in 1986, when the first C-MRS conference was held in Chongqing in October. Various multidisciplinary and interdisciplinary conferences in materials research were first organized through the Chinese Joint Committee of Societies of Materials (CJCSM), composed of 27 societies involved in materials research. As early as 1985, plans were that the joint committee would be the first step towards the formation of a new society-C-MRS.

The name "C-MRS" has been used since the inception of this idea, but it was on May 16, 1991, that C-MRS, an independent interdisciplinary society, was formally established in Beijing. Embracing a broad spectrum of professions and scientific fields, 118 C-MRS council members gath- ered to celebrate the occasion, discuss the society's statutes, and elect officers.

C-MRS is served completely by volunteers who serve the society in their spare time.

A number of immediate tasks have been identified, some already completed:

- To set up an office. The address is CMRS, 7, Baishiqao Road, Beijing 100081, China; fax 1-8412889.

- To publish two journals, one in Chinese, one in English. Plans are to co-publish the English journal with another society.

- To organize conferences in 1991. Already in the planning are "Advanced Materials Research for Automobile Applications," April 1992 in Hubei; and the "Annual CMRS Conference," December 1992 in Guangzhou. C-MRS also plans to cosponsor and work closely with the Chinese Society of Metals to organize the First Pacific Rim International Conference on Advanced Materials and Processing, June

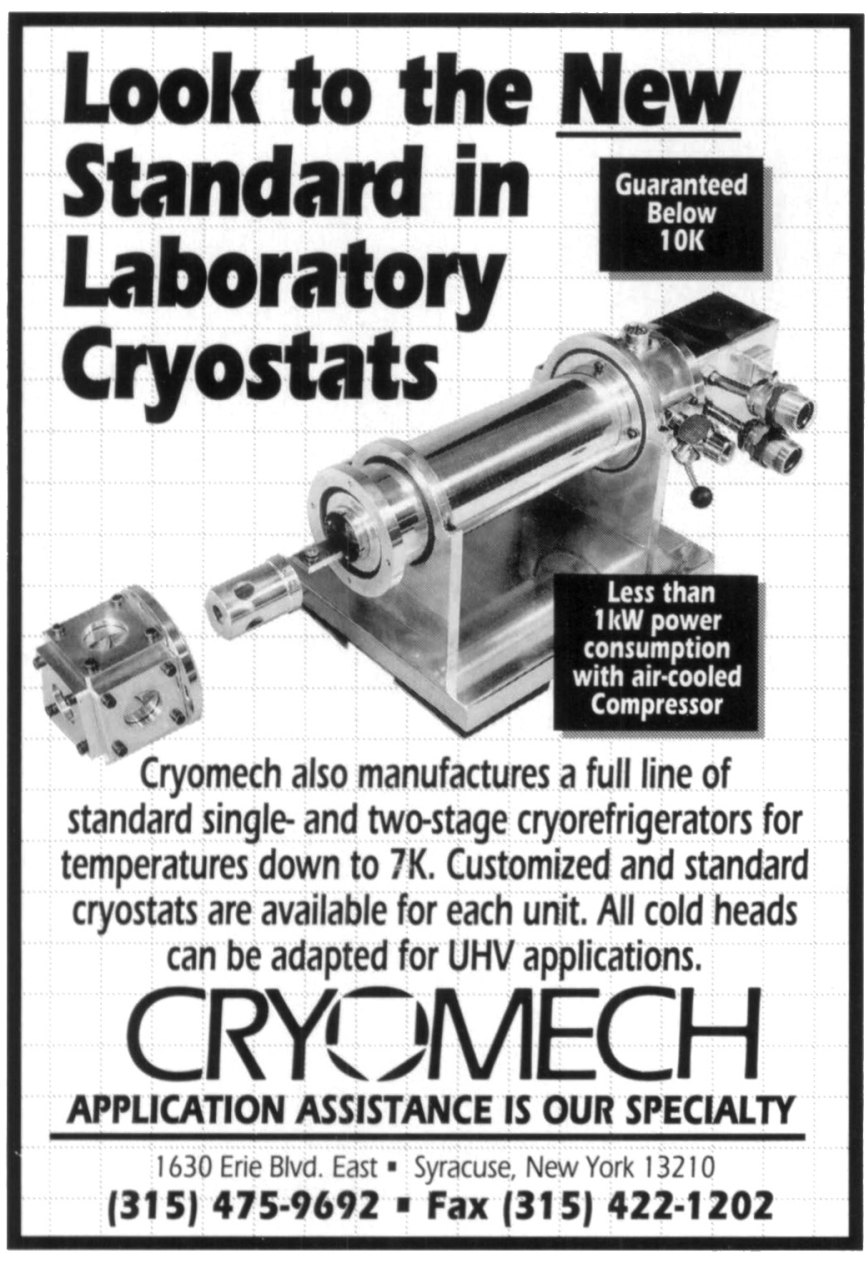

Circle No. 36 on Reader Service Card.

Please visit Booth No. 508 at the MRS Equipment Exhibit in Boston, December 3-5, 1991.
23-27, 1992 in Hangzhou. (See Calendar listing in this issue.)

- To raise funds so the society will be selfsupporting.

Already organized this past February was a Chinese Committee, CC-IUMRS, with Hengde $\mathrm{Li}$ as chairman. One of the functions of CC-IUMRS is to coordinate participation of Chinese materials-related societies in activities of the International Union of Materials Societies.

\section{C-MRS Officers 1991-1994}

President

Hengde Li

Tsinghua University, Beijing

Vice Presidents

Lilan Zhu

State Council of Science and Technology,

Beijing

Fosong Wang

Chinese Academy of Sciences

Hesun Zhu

Beijing Institute of Science and

Technology

Renjie Wu

Shanghai Jiaotong University

Ruisheng Zhang

Ministry of Machinery and Electronics

Jinkun Guo

Shanghai Institute of Ceramics

Chair, Organizing Committee

Ruisheng Zhang

Chair, External Affairs Committee

Hesun Zhu

Chair, Program Committee

Renjie Wu

Chair, Publications Committee

Yiyi Li

Institute of Metals, Shenyang

For a list of Officers and Adhering Bodies of the International Union of Materials Research Societies, see p. 2 in this issue. 


\section{FALL MEETING SYMPOSIUM PROCEEDINGS}

Place your order now for proceeedings from the 1991 MRS Fall Meeting in Boston and you'll receive your books as soon as they are published. SPECIAL PRE-MEETING PRICES EFFECTIVE UNTLL DECEMBER 15, 1991. After that, call MRS for prices.

To order, contact the Materials Research Society, 9800 McKnight Road, Pittsburgh, PA 15237; Phone (412) 367-3012; FAX (412) 367-4373.

Phase Formation and Modification by Beam-Solid Interactions

(Symp. A)

Editors: G.S. Was, L.E. Rehn, D. Follstaedt

ISBN: 1-55899-129-8

$\$ 37.00$ MRS Members

$\$ 44.00$ U.S. List

Volume: 235B

850.00 Foreign

Photons and low Energy Particles in Surface

Processing

(Symp. B)

Editors: C. Ashby, J.H. Brannon, S. Pang

ISBN: 1-55899-130-1

$\$ 35.00$ MRS Members

Volume: $236 \mathrm{~B}$

$\$ 40.00$ U.S. List

$\$ 49.00$ Foreign

Interface Dynamics and Growth

(Symp. Ca)

Editors: K.S. Liang, M.P. Anderson, R.F. Bruinsma, G. Scoles

ISBN: 1-55899-131-X

$\$ 37.00$ MRS Members

$\$ 44.00$ U.S. List

Volume: $237 \mathrm{~B}$

$\$ 50.00$ Foreign

Structure \& Properties of Interfaces in Materials (Symp. Cb)

Editors: W.A.T. Clark, C. L. Briant, U. Dahmen

ISBN: $1-55899-132-8$

Volume: $238 \mathrm{~B}$

$\$ 38.00$ MRS Members

$\$ 54.00$ Foreign

$\$ 47.00$ U.S. List

\section{ical}

Properties III

(Symp. D)

Editors: W.D. Nix, J.C. Bravman, E. Arzt, L.B. Fruend

ISBN: 1-55899-133-6

$\$ 35.00$ MRS Members

$\$ 47.00$ U.S. List

Volume: $239 \mathrm{~B}$

Advanced III-V Compound Semiconductor

Growth, Processing and Devices

(Symp. E)

Editors: S.J. Pearton, D.K. Sadana, J.M. Zavada

ISBN: 1-55899-134-4

$\$ 35.00$ MRS Members

$\$ 47.00$ U.S. List

Volume: $240 \mathrm{~B}$

Low Temperature (LT) GaAs and Related

Materials

(Symp. F)

Editors: G.L. Witt, R. Calawa, U. Mishra, E. Weber

ISBN: 1-55899-135-2

$\$ 35.00$ MRS Members

$\$ 44.00$ U.S. List
Wide Band-Gap Semiconductors

(Symp. G)

Editors: T.D. Moustakas, J.I. Pankove, Y. Hamakawa

ISBN: 1-55899-136-0

Volume: $242 \mathrm{~B}$

$\$ 35.00$ MRS Members

$\$ 44.00$ U.S. List

$\$ 50.00$ Foreign

Ferroelectric Thin Films il

(Symp. I)

Editors: A.I. Kingon, E. R. Myers, B. Tuttle

ISBN: $1-55899-137-9$

\$35.00 MRS Members

Volume 243B

$\$ 40.00$ U.S. Lis

$\$ 49.00$ Foreign

Op̈tical Waveguide Materials

(Symp. J)

Editors: M.M. Broer, H. Kawazoe, G.H. Sigel, R. Th. Kersten

ISBN: 1-55899-138-7

Volume: $244 \mathrm{~B}$

$\$ 35.00$ MRS Members

$\$ 47.00$ U.S. List

$\$ 53.00$ Foreign

Advanced Cementitious Systems: Mechanisms

and Properties

(Symp. K)

Editors: F.P. Glasser, P.L. Pratt, T.O. Mason, J.F. Young, G.J. Mocarthy

ISBN: 1-55899-139-5

$\$ 37.00$ MRS Members

$\$ 44.00$ U.S. List

Volume: $245 \mathrm{~B}$

Shape-Memory Materials and Phenomena -

Fundamental Aspects and Applications

(Symp. M)

Editors: C.T. Liu, M. Wuttig, K. Otsuka, H. Kunsmann

ISBN: $1-55899-140-9$

$\$ 33.00$ MRS Members

$\$ 38.00$ U.S. List

Volume 246B

Organic Solid State Materials

(Symp. N)

Editors: L. Y. Chiang, A.F Garito, D.J. Sandman

ISBN: 1-55899-141-7

$\$ 38.00$ MRS Members

$\$ 44.00$ U.S. List

Volume: $247 \mathrm{~B}$

Complex Fluids

(Symp. 0)

Editors: D. Weitz, E. Sirota, T. Witten, J. Israelachvili

ISBN: 1-55899-142-5

$\$ 35.00$ MRS Members

$\$ 40.00$ U.S. List
Synthesis and Processing of Ceramics:

Scientific Issues

(Symp. Q)

Editors: W.E. Rhine, T.M. Shaw, R.J. Gottschall, $Y$. Chen

ISBN: 1-55899-143-3

$\$ 37.00$ MRS Members

$\$ 42.00$ U.S. List

Volume: $249 \mathrm{~B}$

$\$ 49.00$ Foreign

Chemical Vapor Deposition of Refractory Metals and Ceramics II

(Symp. R)

Editors: T.M. Besmann, B.M. Gallois, J. Warren

ISBN: 1-55899-144-1

$\$ 33.00$ MRS Members

Volume: $250 \mathrm{~B}$

\$38.00 U.S. List

$\$ 44.00$ Foreign

Gas Pressure Effects on Materials Processing

and Design

(Symp. S)

Editors: K. Ishizaki, J.K. Tien, E. Hodge

ISBN: 1-55899-145-X

$\$ 33.00$ MRS Members

$\$ 38.00$ U.S. List

Volume: 25IB

Tissue-Inducing Biomaterials

(Symp. T)

Editors: M. Flanagan, L. Cima, E. Ron

ISBN: 1-55899-146-8

$\$ 37.00$ MRS Members

$\$ 45.00$ U.S. List

Volume: 252B

Application of Multiple Scattering Theory to

Materials Science

(Symp. V)

Editors: W.H. Butler, P.H. Dederichs, A. Gonis, R. Weaver

ISBN: 1-55899-147-6

$\$ 37.00$ MRS Members

Volume: 253B

$\$ 45.00$ U.S. List

$\$ 53.00$ Foreign

Specimen Preparation for Transmission

Electron Microscopy of Materials III

(Symp. W)

Editors: R. Anderson, J. Bravman, B. Tracy

ISBN: 1-55899-148-4

$\$ 35.00$ MRS Members

Volume: $254 \mathrm{~B}$

$\$ 42.00$ U.S. List

$\$ 49.00$ Foreign

Hierarchically Structured Materials

(Symp. Z)

Editors: I.A. Aksay, E. Baer, M. Sarikaya, D.A. Tirrell

ISBN: 1-55899-149-2

\$35.00 MRS Members

$\$ 42.00$ U.S. List

Volunic: 255B

$\$ 49.00$ Foreign

In Europe, Africa, or the Middle East, contact: Clarke Associates - Europe, Ltd., 13a Small Street, Bristol BSI 1DE, England; Phone: 0272268864 ; FAX 0272226437. 